\title{
Editorial for the "Precision Medicine-based Drug Treatment Individualization in Oncology" themed issue Precision Medicine-based Drug Treatment Individualization in Oncology - THEMED ISSUE EDITORAL
}

\author{
Jennifer Martin ${ }^{1}$ and Ian Olver ${ }^{2}$ \\ ${ }^{1}$ University of Newcastle \\ ${ }^{2}$ University of Adelaide
}

June 23, 2020

Understanding of the term 'Precision Medicine' is variable. For clinicians, pharmacists and clinical pharmacologists, it refers to the right decision (treat or not), right drug, right combination, right timing and right dose, taking into account the clinical trial data for the patient group being treated, and finessing that to the individual biological and pharmacological variables either evident, or likely to be evident based on knowledge about comorbidity and body size.

To scientists, precision medicine can mean either developing a new molecule to fit a specific target or cell of interest, or 'finding' based on mathematical and chemical profiling existing drugs that might 'fit' the target of interest (1). However, this focus on 'targets' as the method to improve precision has seemingly ignored the well-known principles of radiobiology, cancer biology and pharmacology, and has not delivered the improved health outcomes expected (2). Similarly, since 2015, U.S. and other government's multi-billiondollar investments into 'precision' drugs "delivering the right treatments, at the right time, every time to the right person whilst helpful to understand some aspects of disease pathophysiology, has also fueled this single target focus $(3)$.

In this Themed issue discussion on aspects of science and medicine people refer to as precision medicine approach are covered. Although varied, much discussion is related to the importance of understanding the way each individual both handles and responds to a drug, and specific dose. How to implement individualized dosing into practice however can be an even more challenging area, with different levels of precision costing different amounts, with a spectrum of clinical and economic benefits.

Added to this difficulty is the systematizing of regimen and dose for specific cancers which have come into oncology practice over the last few years. Moynihan et al argue that the financial dependence of research on industry funding creates a "sponsorship bias" that overplays efficacy and underplays toxicity. This was confirmed in a systematic review comparing industry sponsored with independently funded trials (4).

This is not just an issue with interpretation of results but goes back to trial design. The choice of a comparator may make the outcome of the study drug more favorable (4). Over the years in cancer trials, there has certainly been a move from the principle of treating until maximum response and then allowing a patient time without symptoms or side effects of treatment to studies which are designed to continue treatment until relapse or unacceptable toxicity. This maximizes drug use but is there evidence that prolonging use of a drug maximizes outcomes? 
Maintenance strategies in metastatic disease can be simply continuing the drug used in induction or switching to another drug, which really can be considered as early second line treatment (5). Although established in lymphomas, randomized studies of continuous maintenance therapy in non-small cell lung cancer (NSCLC) led to no improvement in response or survival and similarly for survival in colorectal cancer $(5,6)$. Prolonging first line therapy in breast cancer only showed a marginal survival benefit. There are ongoing studies, but prolonging induction therapy lacks the evidence for widespread adoption. Switching to another drug or targeted therapy, as an early second line treatment, has shown prolonged survival over induction alone in several tumour types (5).

The duration of targeted therapies provides examples of how trial design influences practice. The initial studies of trastuzumab in adjuvant breast cancer initially reported at the American Society of Clinical Oncology in 2005 were designed to give 12 months of therapy and that became the standard of care (7). An ongoing study at the time was comparing 12 months with 24 months. (8). However, an independent French group looked at 6 months compared to 12 months and it is only in 2019 that the final analysis could not show non-inferiority of 6 months of treatment (9). Meanwhile the FinHER study in Finland showed the efficacy and cost-effectiveness of only 9 weeks of adjuvant trastuzumab after chemotherapy $(10,11)$.

In terms of the dose given, the current common method of dosing cytotoxic drugs is based on a patient's body surface area (BSA). This can be inaccurate with considerable variation between patients because patient-related factors such as organ function, age, gender, activity of metabolizing enzymes, drug resistance and concomitant drugs can influence the pharmacokinetics and pharmacodynamics (12). This gives rise to pharmacologically-based dosing being explored to make individual patient dosing more precise.

The first step, however, in the treatment of cancer is to select the drug or drugs most likely to be effective. In the era of precision medicine what is being investigated is identifying mutations in genes or changes in the expression of genes or proteins specific to a tumour, which can be targeted by therapeutics (13). Detecting multiple genomic changes has been made possible by technological advances like next generation sequencing (NGS) replacing older single gene testing. Use multiple platforms combining sequencing of DNA with RNA sequencing and with the more established techniques such as immunohistochemistry (IHC) maximizes the potential to discover druggable targets (14). IHC detects changes at the protein level that reflect gene amplifications such as HER-2 in breast cancer, gastric and colorectal cancer which can be targeted with trastuzumab. Rearrangements include EML4-ALK translocation in non-small cell lung cancer which can be targeted by drugs such as crizotinib. Now there is testing for biomarkers related to PD-L1 expression in various tumours which can be targeted by checkpoint inhibitors such as atezolizumab.

Molecular profiling which allows matching treatments for cancers to their targets has resulted in a boost for new drug development in rare cancers by using drugs targeted to molecular biomarkers that they have in common with more common cancers, in basket trials (15).

Limitations of gene expression profiling and IHC can be illustrated in diffuse large B-cell lymphoma (DLBCL) as summarized by Ofori et al in this issue of the Journal (16). Its subtypes defined by the cell of origin can predict survival and response to chemotherapy, and were initially classified by gene expression profiling. However, fresh tissue had to be available and often only major centers had the capability. IHC methods were subject to observer error. The other issue was that serial tumour profiling during treatment may be able to detect emerging resistance but serial biopsies may not be feasible and surveillance post treatment was only available by imaging, which has not been shown to be associated with a survival benefit.

Liquid biopsies have the potential for solving these issues. Biomarkers in blood or other body fluids can be identified, including from circulating tumour DNA (ctDNA), circulating tumour cells (CTC) or exosomes. Circulating tumour DNA fragments are shed from tumour cells and show mutations and methylation profiles of the tumour which could be used to identify targets or predict recurrence in tumours such as DLBCL (17).

Circulating tumour cells are derived from primary tumours or their metastases and either actively or passively enter the circulation. Their DNA, RNA and proteins could be used to discover the molecular profile of the tumour and their numbers can correlate with treatment outcome (16). De Souza et al have identified 
technological and interpretive challenges to overcome before CTCs are used routinely in the clinic (18).

Exosomes, formed when a cell membrane buds off with contents including protein, nucleic acids, sugars and lipids are taken up by other cells and represent communication between cells. They can circulate in many body fluids. The potential advantage of exomes is their abundance in the fluids and their contents that may reveal multiple biomarkers which in a disease such as DLBCL could be used to subtype and therefore predict prognosis, be used as surveillance during therapy and reveal resistance mechanisms. They could then be used to follow-up post treatment (16).

A further tool to guide dosing of anti-cancer drugs and predicting toxicity and response prior to their administration is pharmacogenomics reviewed by Carr et al (19). Examples with the strongest evidence are assaying for dihydropyrimidine dehydrogenase (DPYD) which is the rate limiting enzyme for 5-FU metabolism, encoded by a gene with multiple variants. Identifying the variant alleles of thiopurine methyltransferase (TMPT) can identify a low activity genotype which metabolizes 6-mercaptopurine to an inactive mercaptopurine resulting in less metabolism of 6-MP to toxic thioguanine nucleotide metabolites. There are many other potential applications of pharmacogenomics, but with equivocal or less evidence. The more widespread use of NGS will allow easier identification of rarer mutations associated with adverse drug reactions. The lack of routine use of pharmacogenomics is multifactorial including the expense, accessibility, the time for processing and the complex interactions including between genomics, clinical factors and the microbiome which account for the individual variations (19).

Personalised drug dosing is important in oncology to prevent overdosing, which otherwise may only become evident when a patient develops severe side effects, or underdosing resulting in lack of efficacy, which may not be revealed until scans show a lack of tumour response. The evidence for some drugs that drug exposure is related to efficacy and toxicity allows for therapeutic dose monitoring (TDM) such as is used for dosing antimicrobials. Some examples of attempting TDM with cytotoxics illustrate the challenges.

For intravenous 5-FU while DPYD genotyping is useful, more precision is needed for bolus and infusional regimens in a variety of cancers, including head and neck and colorectal cancer. In articulating the importance of TDM dosing Schneider JJ et al. in this themed issue, reiterate that 5-FU dosing by BSA only results in 20-30\% patients achieving the therapeutic range. Exploring the relationship between 5 -FU area under the curve (AUC) and a target dose resulted in the recommendation of a therapeutic exposure range of 20$30 \mathrm{mgh} / \mathrm{L}$ for 46 -hour infusion schedules (20). Unfortunately, data is lacking to apply TDM dosing to the oral prodrug capecitabine, which is just as effective as 5-FU but better tolerated.

Other common cytotoxic drugs are more problematic. Muth et al reviewed the taxanes; paclitaxel, docetaxel, nab-paclitaxel and cabazitaxel which illustrate some of the complexities of TDM dosing (21). Paclitaxel which is commonly dosed weekly or 3 weekly has non-linear pharmacokinetics, undergoes hepatic metabolism and biliary excretion and there are interactions with its solvent cremophor, however the time above a plasma concentration of $0.05 \mu \mathrm{mol} / \mathrm{L}$ does predict neutropenia and polyneuropathy and may be associated with a favourable clinical outcome, making TDM dosing desirable. Docetaxel, is also extensively metabolised in the liver, has linear kinetics, but is formulated with polysorbate 80 rather than cremophor. Weekly docetaxel has a more favourable toxicity profile that 3-weekly dosing but it is AUC that predicts febrile neutropenia, mucositis and diarrhoea. Less research has been done than with paclitaxel but a small randomized study of TDM and target concentration intervention (TCI) compared to BSA didn't show a clear advantage for TDM and TCI for docetaxel (22). Unfortunately, is no prospective TDM data for carbazitaxel or nab-paclitaxel.

Early in the development of carboplatin the relationship between drug exposure and efficacy and toxicity was established and dosing was more accurately based on renal function (glomerular filtration rate- GFR) than BSA. However, for specific groups such as infants, anephric patients and those receiving high-dose carboplatin, TDM dosing is more desirable that dosing based on (GFR) as summarized by Barnett S et al (23).

For TDM to be translated into clinical practice, the evidence base must expand, and sampling strategies need to be simplified, perhaps by micro sampling such as using dried blood spots or using body fluids other than 
blood. There must be better access to TDM laboratories, and the provision of clinical decision support for interpreting the results of pharmacometrics which use Bayesian estimations to combine pharmacokinetics, individual patient characteristics and drug concentrations (24).

Finally, a barrier which must be addressed to allow clinical translation of TDM is the demonstration of its economic efficacy which Vithanachchi DT et al present in a descriptive review (25). They reviewed 11 studies and noted that only a few drugs have been studied. However, all studies reviewed found TDM to be cost effective, based on established incremental cost-effectiveness ratios. In future newer therapeutics should have an economic analysis of TDM, incorporating the associated clinical evidence, which in the short term is reduced toxicity and the long term, a survival advantage.

REFERENCES1. Martin JH, Bowden NA. Drug Repurposing - Overcoming the translational hurdles to clinical use. Pharmacol Res Perspect 2019, Nov 26, https://doi.org/10.1002/prp2.548.

2. Fay M, Head R, Martin J. Where is the radiobiology and pharmacology research to improve outcomes on glioblastoma? J Neurooncol 2015, 124: 1-3.

3. Obama B. The Precision Medicine Inititive https://obamawhitehouse.archives.gov/precision-medicine [Last Accessed 11 Dec 2020]

4. Moynihan R, Beros L, Hill S et al. Pathways to independence: towards producing and using trustworthy evidence. BMJ 2019, Dec 3; 367:16576. Doi: 10.1136/bmj.e3502.

5. Rowinski E, Fournel P, Bernichon E, Bouleftour W, Magné N, Mery B. Maintenance therapy in metastatic solid tumours. Innovative strategy or simply second-line treatment? Am J Clin Oncol 2019, 42: 615-623.

6. von Plessen C, Bergman B, Anderson O et al. Palliative chemotherapy beyond three courses conveys no survival or consistent quality-of-life benefits in advanced non-small-cell lung cancer.Br J Cancer 2006, 95: 966-973

7. Perez EA, Romond EH, Suman VJ et al. Four-year follow-up of trastuzumab plus adjuvant chemotherapy for operable human epidermal growth factor receptor positive breast cancer: joint analysis of data from NCCTG N9831 and NSABP B-31. J Clin Oncol 2011, 29: 3366-3372.

8. Cameron D, Piccart-Gebhart MJ, Gelber RD et al. 11 years' follow-up of trastuzumab after adjuvant chemotherapy in HER2-postive early breast cancer: final analysis of the HERceptin Adjuvant (HERA trial. Lancet 2017, 389: 1195-1205.

9. Pivot X, Romieu G, Debled M et al. 6 months versus 12 months of adjuvant trastuzumab in early breast cancer (PHARE): final analysis of a multicenter, open-label, phase 3 randomized trial. Lancet 2019, 393: 2591-2598.

10. Joensuu H, Kellokumpu-Lehtinen PL, Bono P et al. Adjuvant docetaxel or vinorelbine with or without trastuzumab for breast cancer. N Engl JK Med 2006, 354: 809-820.

11. Purmonen TT, Pänkäläinen E,Turunen JH, Asseburg C, Martikainen JA. Short-course adjuvant trastuzumab therapy in early stage breast cancer in Finland: cost-effectiveness and value of information analysis based on the 5-year follow-up results of the FinHer trial. Ann Oncol 2011, 50: 344-352.

12. Kaestner SA, Sewell GJ. Chemotherapy dosing part I: scientific basis for current practice and use of body surface are. Clin Oncol (R Coll Radiol) 2007, 19: 23-37.

13. Malone ER, Oliva M, Sabatini PJB, Stockley TL, Siu LL. Molecular profiling for precision cancer therapies. Genome Med 2020 12:8 https://doi.org/10.1186/s13073-019-0703-1. [Last accessed Jun 10 2020].

14. Zimmer K, Kochner F, Spizzo G, Salem M, Gastl G, Seeber A. Treatment according to molecular profiling in relapsed/refractory cancer patients: a review focusing on latest profiling studies. Comput Struct Biotechnol J 2019, 17: 447-453. 
15. Wang S, Chen R, Tang $\mathrm{Y}$ et al. Comprehensive genomic profiling of rare tumour: routes to targeted therapies. Front Oncol 2020, https://doi.org/10.3389/fonc.2020.00536 [Last accessed 10 Jun 2020]

16 Ofori K, Bhagat G, Rai A. Exosomes as liquid biopsy biomarkers in diffuse large B-cell lymphoma (DLBCL) - Current. State-of-the -art and unmet needs. Br J Clin Pharmacol 2020, X: XX-XX

17. Roschewski M, Dunleavy K, Pittluga S et al. Circulating DNA and CT monitoring in 124 patients with untreated diffuse large B-cell lymphoma: a correlative biomarker study. Lancet Oncol 2015, 16: 541-549.

18. De Souza P, Po J, Scott K et al. Can on study changes in circulating tumour cell (CTC) counts be used as a predictive measure for therapeutic efficacy in caner clinical trials? A review of the literature. Br J Clin Pharmacol 2020, X: XX-XX.

19 Carr DF, Turner RM, Pirmohamed M et al. Pharmacogenomics of anticancer drugs: personalising the choice and dose to manage drug response. Br J Clin Pharmacol 2020, XX: XX-XX.

20. Schneider J, Galettis P, Martin J. Overcoming barriers to implementing precision dosing with 5Fluorouracil and capecitabine. Br J Clin Pharmacol 2020, X:XX-XX.

21 Muth M, Ojara FW, Joerger M. Role of TDM-based dose adjustments for major taxane anticancer drugs. Br J Clin Pharmacol 2020, X: XX-XX.

22. Engel FK, Loos WJ, van der Bol JM et al. Therapeutic drug monitoring for the individualization of docetaxel dosing: a randomised pharmacokinetic study. Clin Cancer Res 2011, 17: 353-362.

23 Barnett S, Kong J, Makin G, Veal GJ. Over a decade of experience with carboplatin therapeutic drug monitoring in a childhood cancer setting in the United Kingdom. Br J Clin Pharmacol 2020, X: XX-XX.

24 Menz BD,, Stocker SL, Verougstraete N et al. Barriers and opportunities for the clinical implementation of therapeutic drug monitoring in oncology. Br J Clin Pharmacol 2020, X: XX-XX.

25. Vithanachchi D, Maujean A, Downes MJ, Scuffham P. A systematic review of economic evaluations of therapeutic drug monitoring interventions for cancer treatments. Br J Clin Pharm 2020, X: XX-XX. 